\title{
A Facile and Efficient Direct Aldol Addition of Simple Thioesters
}

\author{
Julianne M. Yost, Guoqiang Zhou and Don M. Coltart \\ Department of Chemistry and the Center for Chemical Biology, Duke University, \\ Durham, NC 27708
}

\section{Supporting Information}

General Considerations: Unless stated to the contrary, where applicable, the following conditions apply: Reactions were carried out using dried solvents (see below) and under a slight static pressure of Ar (pre-purified quality) that had been passed through a column $(5 \times 20 \mathrm{~cm})$ of Drierite. Glassware was dried in an oven at $120^{\circ} \mathrm{C}$ for at least $12 \mathrm{~h}$ prior to use and then either cooled in a dessicator cabinet over Drierite or assembled quickly while hot, sealed with rubber septa, and allowed to cool under a stream of Ar. Reactions were stirred magnetically using Teflon-coated magnetic stirring bars. Teflon-coated magnetic stirring bars and syringe needles were dried in an oven at $120^{\circ} \mathrm{C}$ for at least 12 $\mathrm{h}$ prior to use and then cooled in a dessicator cabinet over Drierite. Hamilton microsyringes were dried in an oven at $60{ }^{\circ} \mathrm{C}$ for at least $24 \mathrm{~h}$ prior to use and cooled in the same manner. Commercially available Norm-Ject disposable syringes were used. Dry benzene, toluene, $\mathrm{Et}_{2} \mathrm{O}, \mathrm{CH}_{2} \mathrm{Cl}_{2}, \mathrm{THF}, \mathrm{MeCN}$ and DME were obtained using an Innovative Technologies solvent purification system. All other dry solvents were of anhydrous quality purchased from Aldrich. Commercial grade solvents were used for routine purposes without further purification. $\mathrm{Et}_{3} \mathrm{~N}$, pyridine, $i$ - $\mathrm{Pr}_{2} \mathrm{NEt}$, 2,6-lutidine, $i$ $\mathrm{Pr}_{2} \mathrm{NH}$ and TMEDA were distilled from $\mathrm{CaH}_{2}$ under a $\mathrm{N}_{2}$ atmosphere prior to use. Flash column chromatography was performed on silica gel $60\left(230-400\right.$ mesh). ${ }^{1} \mathrm{H}$ and ${ }^{13} \mathrm{C}$ NMR were recorded on a $300 \mathrm{MHz}$ spectrometer at ambient temperature. All ${ }^{1} \mathrm{H}$ chemical shifts are reported in ppm $(\delta)$ relative to TMS; ${ }^{13} \mathrm{C}$ shifts are reported relative to the corresponding NMR solvent.

The following reaction is representative of those depicted in Table 1:

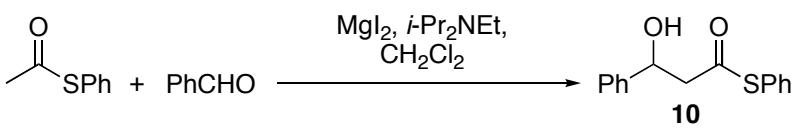

$\boldsymbol{\beta}$-Hydroxy thioester (10). $\mathrm{MgI}_{2}(0.167 \mathrm{~g}, 0.6 \mathrm{mmol})$ was added to a stirred solution of thioester $5(0.076 \mathrm{~g}, 0.5 \mathrm{mmol})$ and benzaldehyde $(61 \mu \mathrm{L}, 0.6 \mathrm{mmol})$ in $\mathrm{CH}_{2} \mathrm{Cl}_{2}(2.5 \mathrm{~mL})$, followed by the addition of $i-\operatorname{Pr}_{2} \mathrm{NEt}(0.11 \mathrm{~mL}, 0.65 \mathrm{mmol})$. Stirring was continued for 30 min and EtOAc $(2.5 \mathrm{~mL})$ and $10 \%(\mathrm{v} / \mathrm{v})$ aqueous $\mathrm{HCl}(2.5 \mathrm{~mL})$ were added. Stirring was continued for $15 \mathrm{~min}$ and the mixture was partitioned between EtOAc $(15 \mathrm{~mL})$ and $\mathrm{H}_{2} \mathrm{O}(2 \mathrm{~mL})$. The aqueous phase was extracted with EtOAc $(3 \times 5 \mathrm{~mL})$ and the combined organic extracts were washed with saturated aqueous $\mathrm{NaCl}$, dried $\left(\mathrm{MgSO}_{4}\right)$, and evaporated to give a light-yellow solid. Flash chromatography over silica gel, using 10:90 EtOAc-hexanes gave $\mathbf{1 0}(0.121 \mathrm{~g}$; $94 \%)$ as a pure, colorless solid: ${ }^{1} \mathbf{H}$ NMR 
$\left(\mathrm{CDCl}_{3}, 300 \mathrm{MHz}\right): \delta$ 7.50-7.26 (m, 10H), 5.21 (X of an ABX system, apparent td, $J=$ 3.4, $8.7 \mathrm{~Hz}, 1 \mathrm{H}$ ), 3.12 (A of an ABX system, apparent dd, $J=8.8,16.0 \mathrm{~Hz}, 1 \mathrm{H}$ ), 3.03 (B of an ABX system, apparent dd, $J=3.8,16.0 \mathrm{~Hz}, 1 \mathrm{H}), 2.97(\mathrm{~d}, J=3.3 \mathrm{~Hz}, 1 \mathrm{H}) ;{ }^{13} \mathbf{C}$ NMR $\left(\mathrm{CDCl}_{3}, 300 \mathrm{MHz}\right): \delta 197.4,142.3,134.6,129.8,129.4,128.8,128.1,127.2$, 125.8, 70.9, 52.2; ESI-MS $m / z$ calcd for $\mathrm{C}_{15} \mathrm{H}_{14} \mathrm{NaO}_{2} \mathrm{~S}: 281.1$, found: 280.8 .

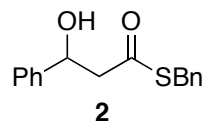

$\boldsymbol{\beta}$-Hydroxy thioester (2). Flash chromatography over silica gel, using 10:90 EtOAchexanes gave $2(0.1294 \mathrm{~g} ; 95 \%)$ as a pure, colorless solid: ${ }^{1} \mathbf{H}$ NMR $\left(\mathrm{CDCl}_{3}, 300 \mathrm{MHz}\right)$ : $\delta$ 7.42-7.18 (m, 10H), 5.20 (X of an ABX system, apparent td, $J=3.6,8.7 \mathrm{~Hz}, 1 \mathrm{H}), 4.17$ and $4.15\left(\mathrm{AB} \mathrm{q}, \Delta v_{\mathrm{AB}}=6.6 \mathrm{~Hz}, J=13.8 \mathrm{~Hz}, 2 \mathrm{H}\right), 3.08-2.88[\mathrm{~m}, 3 \mathrm{H}$, including $\mathrm{A}$ of an ABX system, apparent dd, at $\delta 3.02(J=9.0,15.9 \mathrm{~Hz}, 1 \mathrm{H})$ and $\mathrm{B}$ of an $\mathrm{ABX}$ system, apparent dd, at $\delta 2.93(J=3.9,15.9 \mathrm{~Hz}, 1 \mathrm{H})] ;{ }^{13} \mathbf{C} \mathbf{~ N M R}\left(\mathrm{CDCl}_{3}, 300 \mathrm{MHz}\right): \delta 198.2$, $142.4,137.2,129.0,128.82,128.75,128.1,127.5,125.8,71.0,52.4,33.4$; ESI-MS $\mathrm{m} / \mathrm{z}$ calcd for $\mathrm{C}_{16} \mathrm{H}_{16} \mathrm{NaO}_{2} \mathrm{~S}: 295.1$, found: 294.9.

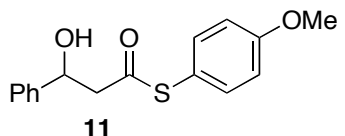

$\boldsymbol{\beta}$-Hydroxy thioester (11). Flash chromatography over silica gel, using 15:85 EtOAchexanes gave $11(0.1413 \mathrm{~g} ; 98 \%)$ as a pure, colorless solid: ${ }^{1} \mathbf{H} \mathbf{~ N M R}\left(\mathrm{CDCl}_{3}, 300 \mathrm{MHz}\right)$ : $\delta$ 7.39-7.20 (m, 7H), 7.00-6.90 (m, 2H), 5.20 (X of an ABX system, apparent td, $J=3.3$, $8.7 \mathrm{~Hz}, 1 \mathrm{H}), 3.83(\mathrm{~s}, 3 \mathrm{H}), 3.14-2.97$ [m, 3H, including A of an ABX system, apparent dd, at $\delta 3.09(J=8.7,15.9 \mathrm{~Hz}, 1 \mathrm{H})$, a d at $\delta 3.04(J=4.2 \mathrm{~Hz})$, and B of an ABX system, apparent dd, at $\delta 2.99(J=3.3,15.9 \mathrm{~Hz}, 1 \mathrm{H})] ;{ }^{13} \mathbf{C} \mathbf{~ N M R}\left(\mathrm{CDCl}_{3}, 300 \mathrm{MHz}\right): \delta 198.7$, $161.0,142.3,136.2,128.8,128.1,125.8,117.8,115.1,70.9,55.5,51.9 ;$ ESI-MS $\mathrm{m} / \mathrm{z}$ calcd for $\mathrm{C}_{16} \mathrm{H}_{16} \mathrm{NaO}_{3} \mathrm{~S}: 311.1$, found: 310.9 .

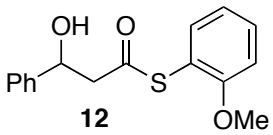

$\boldsymbol{\beta}$-Hydroxy thioester (12). Flash chromatography over silica gel, using 15:85 EtOAchexanes gave $\mathbf{1 2}(0.1384 \mathrm{~g} ; 96 \%)$ as a pure, light-yellow solid: ${ }^{1} \mathbf{H} \mathbf{~ N M R}\left(\mathrm{CDCl}_{3}, 300\right.$ MHz): $\delta$ 7.45-7.20 (m, 7H), 7.06-6.97 (m, 2H), 5.20 (X of an ABX system, apparent dd, $J=8.1,4.5 \mathrm{~Hz}, 1 \mathrm{H}), 3.85(\mathrm{~s}, 3 \mathrm{H}), 3.24-2.96[\mathrm{~m}, 3 \mathrm{H}$, including A of an ABX system, apparent dd, at $\delta 3.15(J=5.4,15.9 \mathrm{~Hz}, 1 \mathrm{H})$, and $\mathrm{B}$ of an $\mathrm{ABX}$ system, apparent dd, at $\delta$ $3.05(J=4.2,15.9 \mathrm{~Hz}, 1 \mathrm{H})] ;{ }^{13} \mathbf{C}$ NMR $\left(\mathrm{CDCl}_{3}, 300 \mathrm{MHz}\right): \delta 197.0,159.2,142.3,136.8$, $132.1,128.7,128.0,125.8,121.3,115.4,111.8,71.0,56.1,52.1$; ESI-MS $m / z$ calcd for $\mathrm{C}_{16} \mathrm{H}_{16} \mathrm{NaO}_{3} \mathrm{~S}: 311.1$, found: 310.9 . 


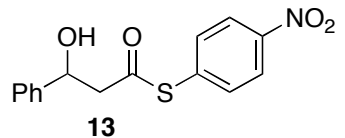

$\boldsymbol{\beta}$-Hydroxy thioester (13). Flash chromatography over silica gel, using 20:80 EtOAchexanes gave $13(0.1395 \mathrm{~g} ; 92 \%)$ as a pure, light-yellow solid: ${ }^{1} \mathbf{H}$ NMR $\left(\mathrm{CDCl}_{3}, 300\right.$ MHz): $\delta$ 8.30-8.20 (m, 2H), 7.70-7.55 (m, 2H), 7.50-7.22 (m, 5H), 5.26 (X of an ABX system, apparent td, $J=3.6,9.0 \mathrm{~Hz}, 1 \mathrm{H}), 3.25-3.00[\mathrm{~m}, 2 \mathrm{H}$, including A of an ABX system, apparent dd, at $\delta 3.19(J=9.0,15.9 \mathrm{~Hz}, 1 \mathrm{H})$, and B of an ABX system, apparent dd, at $\delta 3.06(J=3.6,15.9 \mathrm{~Hz}, 1 \mathrm{H})], 2.64(\mathrm{~d}, J=3.6 \mathrm{~Hz}, 1 \mathrm{H}) ;{ }^{13} \mathbf{C ~ N M R}\left(\mathrm{CDCl}_{3}, 300\right.$ MHz): $\delta$ 194.5, 148.4, 142.1, 135.7, 134.9, 128.9, 128.4, 125.8, 124.2, 70.9, 52.8; ESIMS $m / z$ calcd for $\mathrm{C}_{15} \mathrm{H}_{13} \mathrm{NNaO}_{4} \mathrm{~S}: 326.0$, found: 325.9.

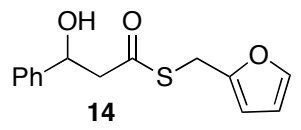

$\beta$-Hydroxy thioester (14). Flash chromatography over silica gel, using 10:90 EtOAchexanes gave $14(0.1259 \mathrm{~g} ; 96 \%)$ as a pure, light-yellow oil: ${ }^{1} \mathbf{H}$ NMR $\left(\mathrm{CDCl}_{3}, 300\right.$ $\mathrm{MHz}): \delta$ 7.41-7.23 (m, 6H), 6.33-6.18 (m, 2H), 5.20 (X of an ABX system, broad apparent td, $(J=2.7,8.7 \mathrm{~Hz}, 1 \mathrm{H}) 4.20$ and $4.17\left(\mathrm{AB} \mathrm{q}, \Delta v_{\mathrm{AB}}=10.4 \mathrm{~Hz}, J=15.3 \mathrm{~Hz}\right.$, $2 \mathrm{H}), 3.09-2.88[\mathrm{~m}, 3 \mathrm{H}$, including $\mathrm{A}$ of an $\mathrm{ABX}$ system, apparent dd, at $\delta 3.03(J=8.8$, $15.8 \mathrm{~Hz}), \mathrm{B}$ of an ABX system, apparent dd, at $\delta 2.93(J=3.9,15.8 \mathrm{~Hz})$, overlapping a broad d at $\delta 2.92(J=2.4 \mathrm{~Hz})] ;{ }^{13} \mathbf{C}$ NMR $\left(\mathrm{CDCl}_{3}, 300 \mathrm{MHz}\right): \delta 197.5,150.1,142.4$, 142.3, 128.7, 128.0, 125.7, 110.7, 108.2, 70.8, 52.4, 25.8; ESI-MS $\mathrm{m} / \mathrm{z}$ calcd for $\mathrm{C}_{14} \mathrm{H}_{14} \mathrm{NaO}_{3} \mathrm{~S}: 285.1$, found: 284.8 .

\section{The following reaction is representative of those depicted in Table 2 and Scheme 2:}

The following reactions were conducted using untreated $\mathrm{CH}_{2} \mathrm{Cl}_{2}$, open to the atmosphere. Glassware and stirring bars were dried as described above, but allowed to cool open to the atmosphere.

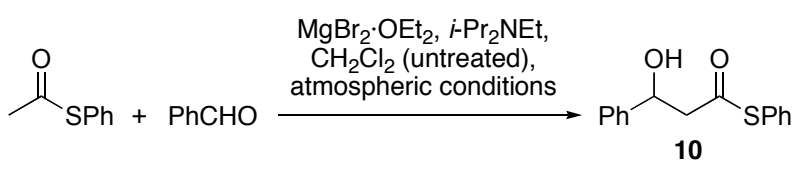

$\beta$-Hydroxy thioester (10). $\mathrm{MgBr}_{2} \cdot \mathrm{OEt}_{2}(0.181 \mathrm{~g}, 0.7 \mathrm{mmol})$ was added to a stirred solution of thioester $5(0.076 \mathrm{~g}, 0.5 \mathrm{mmol})$ and benzaldehyde $(61 \mu \mathrm{L}, 0.6 \mathrm{mmol})$ in $\mathrm{CH}_{2} \mathrm{Cl}_{2}(2.5 \mathrm{~mL})$, followed by the addition of $i$ - $\operatorname{Pr}_{2} \mathrm{NEt}(0.17 \mathrm{~mL}, 1.0 \mathrm{mmol})$. The reaction flask was capped to prevent evaporation. Stirring was continued for $30 \mathrm{~min}$ and then EtOAc $(2.5 \mathrm{~mL})$ and $10 \%(\mathrm{v} / \mathrm{v})$ aqueous $\mathrm{HCl}(2.5 \mathrm{~mL})$ were added. Stirring was continued for $20 \mathrm{~min}$ and the mixture was partitioned between EtOAc $(30 \mathrm{~mL})$ and $\mathrm{H}_{2} \mathrm{O}$ $(2 \mathrm{~mL})$. The aqueous phase was extracted with EtOAc $(3 \times 5 \mathrm{~mL})$ and the combined organic extracts were washed with saturated aqueous $\mathrm{NaCl}$, dried $\left(\mathrm{MgSO}_{4}\right)$, and 
evaporated to give a light-yellow solid. Flash chromatography over silica gel, using 10:90 EtOAc-hexanes gave $\mathbf{1 0}(0.1240 \mathrm{~g} ; \mathbf{9 6 \%})$ as a pure, colorless solid. Spectroscopic data was identical to that reported above.

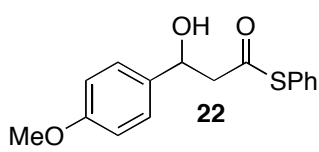

$\boldsymbol{\beta}$-Hydroxy thioester (22). Flash chromatography over silica gel, using 20:80 EtOAchexanes gave $22(0.1399 \mathrm{~g} ; 97 \%)$ as a pure, colorless solid. ${ }^{\mathbf{1}} \mathbf{H} \mathbf{~ N M R}\left(\mathrm{CDCl}_{3}, 300 \mathrm{MHz}\right)$ : $\delta$ 7.44-6.84 [m, 9H, including apparent $\mathrm{d}$ at $\delta 7.30(\mathrm{~J}=8.8 \mathrm{~Hz})$, and apparent $\mathrm{d}$ at $\delta 6.89$ $(J=8.8 \mathrm{~Hz})], 5.16$ (X of an ABX system, broad apparent d $(J=8.7 \mathrm{~Hz}, 1 \mathrm{H}), 3.80(\mathrm{~s}, 3 \mathrm{H})$, 3.11 (A of an ABX system, apparent dd, $J=8.7,15.9 \mathrm{~Hz}, 1 \mathrm{H}$ ), 3.00 (B of an ABX system, apparent dd, $J=3.6,15.9 \mathrm{~Hz}, 1 \mathrm{H}), 2.90$ (broad apparent s, $1 \mathrm{H}) ;{ }^{13} \mathbf{C}$ NMR $\left(\mathrm{CDCl}_{3}, 300 \mathrm{MHz}\right): \delta 197.1,159.3,134.53,134.50,129.7,129.3,127.2,127.0,114.0$, 70.4, 55.3, 52.2; ESI-MS $m / z$ calcd for $\mathrm{C}_{16} \mathrm{H}_{16} \mathrm{NaO}_{3} \mathrm{~S}: 311.1$, found: 310.8 .

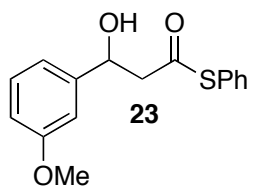

$\boldsymbol{\beta}$-Hydroxy thioester (23). Flash chromatography over silica gel, using 20:80 EtOAchexanes gave $23(0.1384 \mathrm{~g} ; 96 \%)$ as a pure, colorless solid. ${ }^{1} \mathbf{H}$ NMR $\left(\mathrm{CDCl}_{3}, 300\right.$ $\mathrm{MHz}): \delta 7.50-7.22(\mathrm{~m}, 6 \mathrm{H}), 6.98-6.78(\mathrm{~m}, 3 \mathrm{H}), 5.19$ (X of an ABX system, apparent overlapping td, $J=3.6,8.5 \mathrm{~Hz}, 1 \mathrm{H}), 3.81(\mathrm{~s}, 3 \mathrm{H}), 3.17-2.92[\mathrm{~m}, 3 \mathrm{H}$, including A of an ABX system, apparent dd, at $\delta 3.10(J=8.5,16.0 \mathrm{~Hz}), \mathrm{B}$ of an ABX system, apparent dd, at $\delta 3.02(J=3.6,16.0 \mathrm{~Hz})$, and a d at $\delta 2.97(J=3.6 \mathrm{~Hz})] ;{ }^{13} \mathbf{C} \mathbf{N M R}\left(\mathrm{CDCl}_{3}, 300\right.$ MHz): $\delta$ 197.0, 159.8, 144.0, 134.5, 129.7, 129.3, 127.2, 118.0, 113.6, 111.1, 70.6, 55.3, 52.2 (2 peaks overlapping); ESI-MS $m / z$ calcd for $\mathrm{C}_{16} \mathrm{H}_{16} \mathrm{NaO}_{3} \mathrm{~S}: 311.1$, found: 310.8 .

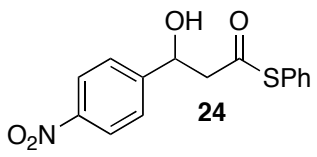

$\beta$-Hydroxy thioester (24). Flash chromatography over silica gel, using 20:80 EtOAchexanes gave $24(0.1355 \mathrm{~g} ; 94 \%)$ as a pure, colorless solid. ${ }^{1} \mathbf{H}$ NMR $\left(\mathrm{CDCl}_{3}, 300\right.$ MHz): $\delta 8.22$ (apparent d, $J=8.7 \mathrm{~Hz}, 2 \mathrm{H}$ ), 7.56 (apparent d, $J=8.7 \mathrm{~Hz}, 2 \mathrm{H}$ ), 7.48-7.34 $(\mathrm{m}, 5 \mathrm{H}), 5.32(\mathrm{dt}, J=3.6,6.3 \mathrm{~Hz}, 1 \mathrm{H}), 3.30(\mathrm{~d}, J=3.6 \mathrm{~Hz}, 1 \mathrm{H}), 3.08(\mathrm{~d}, J=6.3 \mathrm{~Hz}, 2 \mathrm{H})$; ${ }^{13} \mathbf{C}$ NMR $\left(\mathrm{CDCl}_{3}, 300 \mathrm{MHz}\right): \delta 197.3,149.4,147.7,134.6,130.1,129.6,126.7,124.0$, 69.9, 51.6 (2 peaks overlapping); ESI-MS $m / z$ calcd for $\mathrm{C}_{15} \mathrm{H}_{13} \mathrm{NNaO}_{4} \mathrm{~S}: 326.0$, found: 325.8 


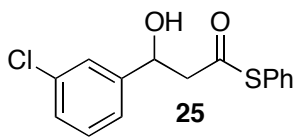

$\boldsymbol{\beta}$-Hydroxy thioester (25). Flash chromatography over silica gel, using 20:80 EtOAchexanes gave $25(0.1420 \mathrm{~g} ; 95 \%)$ as a pure, colorless solid. ${ }^{1} \mathbf{H}$ NMR $\left(\mathrm{CDCl}_{3}, 300\right.$ $\mathrm{MHz}): \delta 7.46-7.36(\mathrm{~m}, 6 \mathrm{H}), 7.34-7.20(\mathrm{~m}, 3 \mathrm{H}), 5.18$ (X of ABX system, apparent overlapping td, $J=3.9,7.8 \mathrm{~Hz}, 1 \mathrm{H}), 3.14-2.96[\mathrm{~m}, 3 \mathrm{H}$, including A of an ABX system, apparent dd, at $\delta 3.08(J=7.8,15.9 \mathrm{~Hz})$, overlapping a d at $\delta 3.08(J=3.3 \mathrm{~Hz})$, and B of an $\mathrm{ABX}$ system, apparent dd, at $\delta 3.01(J=4.4,15.9 \mathrm{~Hz})] ;{ }^{13} \mathbf{C ~ N M R}\left(\mathrm{CDCl}_{3}, 300 \mathrm{MHz}\right)$ : $\delta 197.2,144.3,134.6,130.0,129.8,129.4,128.1,126.9,126.0,123.9,70.1,51.9$ (2 peaks overlapping); ESI-MS $m / z$ calcd for $\mathrm{C}_{15} \mathrm{H}_{13} \mathrm{ClNaO}_{2} \mathrm{~S}: 315.0$, found: 314.8 .

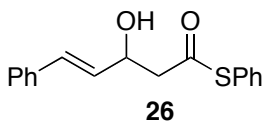

$\boldsymbol{\beta}$-Hydroxy thioester (26). Flash chromatography over silica gel, using 10:90 EtOAchexanes gave 26 (0.1307 g; 92\% with trace impurities) as a colorless solid. ${ }^{1} \mathbf{H}$ NMR $\left(\mathrm{CDCl}_{3}, 300 \mathrm{MHz}\right): \delta 7.46-7.20(\mathrm{~m}, 10 \mathrm{H}), 6.68(\mathrm{~d}, J=15.8 \mathrm{~Hz}, 1 \mathrm{H}), 6.23(\mathrm{dd}, J=6.0$, $15.8 \mathrm{~Hz}, 1 \mathrm{H}), 4.87-4.76(\mathrm{~m}, 1 \mathrm{H}), 3.00$ (apparent d, $J=6.6 \mathrm{~Hz}, 2 \mathrm{H}), 2.74(\mathrm{~d}, J=4.2 \mathrm{~Hz}$, $1 \mathrm{H}) ;{ }^{13} \mathrm{C}$ NMR $\left(\mathrm{CDCl}_{3}, 300 \mathrm{MHz}\right): \delta 197.1,136.4,134.6,131.2,129.8,129.6,129.4$, 128.7, 128.0, 127.2, 126.7, 69.5, 50.3; ESI-MS $\mathrm{m} / z$ calcd for $\mathrm{C}_{17} \mathrm{H}_{16} \mathrm{NaO}_{2} \mathrm{~S}$ : 307.1, found: 306.9 .

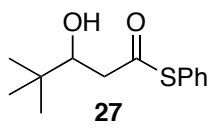

$\boldsymbol{\beta}$-Hydroxy thioester (27). Flash chromatography over silica gel, using 10:90 EtOAchexanes gave $27(0.1120 \mathrm{~g} ; 94 \%)$ as a pure, light-yellow oil. ${ }^{1} \mathbf{H}$ NMR $\left(\mathrm{CDCl}_{3}, 300\right.$ $\mathrm{MHz}$ ): $\delta$ 7.44-7.40 (m, 5H), 3.78 (X of an ABX system, apparent $\mathrm{d}, J=10.2 \mathrm{~Hz}, 1 \mathrm{H}$ ), 2.94-2.58 [m, 3H, including A of an ABX system, apparent dd, at $\delta 2.88(J=2.0,15.8$ $\mathrm{Hz}), \mathrm{B}$ of an ABX system, apparent dd, at $\delta 2.71(\mathrm{~J}=10.2,15.8 \mathrm{~Hz})$, and a d at $\delta 2.62(\mathrm{~J}$ $=3.3 \mathrm{~Hz})], 0.93(\mathrm{~s}, 9 \mathrm{H}) ;{ }^{13} \mathbf{C}$ NMR $\left(\mathrm{CDCl}_{3}, 300 \mathrm{MHz}\right): \delta 198.8,134.6,129.7,129.4$, 127.4, 76.0, 45.9, 34.8, 25.7; ESI-MS $m / z$ calcd for $\mathrm{C}_{13} \mathrm{H}_{18} \mathrm{NaO}_{2} \mathrm{~S}: 261.1$, found: 260.9 .

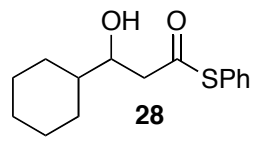

$\boldsymbol{\beta}$-Hydroxy thioester (28). Flash chromatography over silica gel, using 10:90 EtOAchexanes gave $28(0.1084 \mathrm{~g} ; 82 \%)$ as a pure, colorless solid. ${ }^{1} \mathbf{H}$ NMR $\left(\mathrm{CDCl}_{3}, 300\right.$ $\mathrm{MHz}): \delta$ 7.43-7.37 (m, 5H), 3.92-3.78 (X of an ABX system, m, 1H), 2.90-2.64 [m, 3H, including A of an ABX system, apparent dd, at $\delta 2.86(J=3.3,15.9 \mathrm{~Hz}), \mathrm{B}$ of an ABX system, apparent dd, at $\delta 2.77(J=8.7,15.9 \mathrm{~Hz})$, and a d at $\delta 2.68(J=3.9 \mathrm{~Hz})], 1.94-$ 
$0.88(\mathrm{~m}, 11 \mathrm{H}) ;{ }^{13} \mathbf{C}$ NMR $\left(\mathrm{CDCl}_{3}, 300 \mathrm{MHz}\right): \delta 198.3,134.5,129.6,129.3,127.4,72.7$, 47.8, 43.2, 28.9, 28.1, 26.4, 26.2, 26.1; ESI-MS $m / z$ calcd for $\mathrm{C}_{15} \mathrm{H}_{20} \mathrm{NaO}_{2} \mathrm{~S}: 287.1$, found: 286.9.

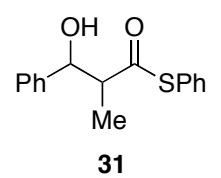

$\boldsymbol{\alpha}$-Methyl- $\boldsymbol{\beta}$-Hydroxy thioester (31). Flash chromatography over silica gel, using 20:80 EtOAc-hexanes gave $31(0.1226 \mathrm{~g} ; 90 \%)$ as a pure, colorless oil comprised of a 2:1 (syn:anti) mixture of diastereomers. ${ }^{1} \mathbf{H}$ NMR $\left(\mathrm{CDCl}_{3}, 300 \mathrm{MHz}\right): \delta 7.52-7.22(\mathrm{~m}, 10 \mathrm{H})$, 5.20-4.78 [m, $1 \mathrm{H}$, including a dd at $\delta 5.13(J=2.7,4.2 \mathrm{~Hz})$ and a dd at $\delta 4.84(J=4.4$, $8.2 \mathrm{~Hz}), 3.20-2.98(\mathrm{~m}, 1 \mathrm{H}), 2.82-2.67(\mathrm{~m}, 1 \mathrm{H}$, including a $\mathrm{m}$ from $\delta 2.82-2.75$ and a $\mathrm{m}$ from $\delta 2.73-2.67), 1.32-1.06[\mathrm{~m}, 3 \mathrm{H}$, including a d at $\delta 1.30(J=7.2 \mathrm{~Hz})$ and a $\mathrm{d}$ at $\delta 1.10$ $(J=7.2 \mathrm{~Hz})] ;{ }^{13} \mathbf{C}$ NMR $\left(\mathrm{CDCl}_{3}, 300 \mathrm{MHz}\right): \delta 201.8,201.6,141.6,141.2,134.52$, $134.50,129.6,129.5,129.25,129.23,128.6,128.3,128.2,127.7,127.5,127.2$, 126.7, 126.2, 76.6, 74.0, 55.3, 55.1, 15.4, 11.9; ESI-MS $m / z$ calcd for $\mathrm{C}_{16} \mathrm{H}_{16} \mathrm{NaO}_{2} \mathrm{~S}: 295.1$, found: 294.8 .

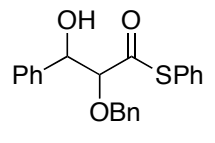

32

$\boldsymbol{\alpha}$-Benzyloxy- $\boldsymbol{\beta}$-hydroxy thioester (32). Flash chromatography over silica gel, using 20:80 EtOAc-hexanes gave $32(0.1768 \mathrm{~g} ; 97 \%)$ as a pure, colorless solid comprised of a 1:1 (syn:anti) mixture of diastereomers. ${ }^{1} \mathbf{H}$ NMR $\left(\mathrm{CDCl}_{3}, 300 \mathrm{MHz}\right): \delta 7.48-7.16(\mathrm{~m}$, $15 \mathrm{H}), 5.13-4.92[\mathrm{~m}, 1 \mathrm{H}$, including a dd at $\delta 5.07(J=4.2,6.6 \mathrm{~Hz})$, and a dd at $\delta 4.95(J=$ $3.9,6.6 \mathrm{~Hz})], 4.80-4.25\left[\mathrm{~m}, 2 \mathrm{H}\right.$, including an $\mathrm{AB} \mathrm{q}$ at $\delta 4.75$ and $4.70\left(\Delta v_{\mathrm{AB}}=16.0 \mathrm{~Hz}, J\right.$ $=11.1 \mathrm{~Hz})$ and an $\mathrm{AB}$ q at $\delta 4.43$ and $\left.4.26\left(\Delta v_{\mathrm{AB}}=24.6 \mathrm{~Hz}, J=11.1 \mathrm{~Hz}\right)\right], 4.22-4.12[\mathrm{~m}$, $1 \mathrm{H}$, including a d at $\delta 4.19(J=4.2 \mathrm{~Hz})$ and a d at $\delta 4.17(\mathrm{~J}=6.6 \mathrm{~Hz})], 3.30-2.90[\mathrm{~m}, 1 \mathrm{H}$, including a dd at $\delta 3.10(J=1.2,3.9 \mathrm{~Hz})$ and a dd at $\delta 2.94(J=1.5,6.6 \mathrm{~Hz})] ;{ }^{13} \mathbf{C} \mathbf{N M R}$ $\left(\mathrm{CDCl}_{3}, 300 \mathrm{MHz}\right): \delta 201.0,199.5,139.4,139.1,136.5,136.2,134.62,134.56,129.43$, $129.40,129.2,128.45,128.42,128.3,128.23,128.16,128.1,128.0,127.4,127.09$, 127.06, 126.4, 88.3, 87.4, 74.9, 74.6, 74.5 (some peaks overlapping); ESI-MS $m / z$ calcd for $\mathrm{C}_{22} \mathrm{H}_{20} \mathrm{NaO}_{3} \mathrm{~S}: 387.1$, found: 386.8 .

The following is representative of the synthesis of thioesters 1, 6 and 7.

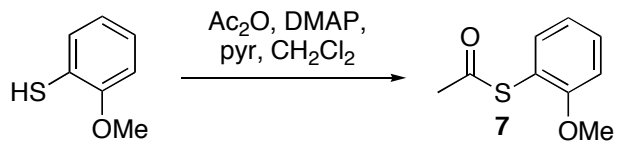

$S$-(2-Methoxy)phenyl thioacetate (7). $\mathrm{Ac}_{2} \mathrm{O}(1.86 \mathrm{~mL}, 19.68 \mathrm{mmol})$ was added via syringe to a stirred solution of the thiol $(2.00 \mathrm{~mL}, 16.43 \mathrm{mmol})$, DMAP $(0.0464 \mathrm{~g}, 0.38$ 
mmol), pyridine $(1 \mathrm{~mL})$ and $\mathrm{CH}_{2} \mathrm{Cl}_{2}(19 \mathrm{~mL})$. The mixture was allowed to stir for $12 \mathrm{~h}$ and then partitioned between EtOAc and saturated aqueous $\mathrm{NaHCO}_{3}$. The organic phase was washed with water, saturated aqueous $\mathrm{NaCl}$, dried $\left(\mathrm{MgSO}_{4}\right)$, and evaporated to give a light-yellow oil. Flash chromatography over silica gel, using 6:94 EtOAc-hexanes gave $7(2.7551 \mathrm{~g} ; 92 \%)$ as a pure, colorless liquid. ${ }^{1} \mathbf{H} \mathbf{~ N M R}\left(\mathrm{CDCl}_{3}, 300 \mathrm{MHz}\right): \delta$ 7.48-7.34 $(\mathrm{m}, 2 \mathrm{H}), 7.04-6.92(\mathrm{~m}, 2 \mathrm{H}), 3.86(\mathrm{~s}, 3 \mathrm{H}), 2.41(\mathrm{~s}, 3 \mathrm{H}) ;{ }^{13} \mathbf{C ~ N M R}\left(\mathrm{CDCl}_{3}, 300 \mathrm{MHz}\right): \delta$ 193.5, 159.2, 136.8, 131.8, 121.2, 116.2, 111.6, 56.0, 30.1; ESI-MS $\mathrm{m} / \mathrm{z}$ calcd for $\mathrm{C}_{9} \mathrm{H}_{10} \mathrm{NaO}_{2} \mathrm{~S}: 205.0$, found: 204.8 .

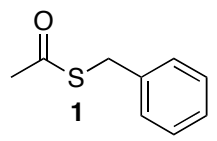

$\boldsymbol{S}$-Benzyl thioacetate (1). Flash chromatography over silica gel, using 8:92 EtOAchexanes gave $1(1.6637 \mathrm{~g} ; 87 \%)$ as a pure, colorless liquid. Spectroscopic data was identical to that reported previously. ${ }^{1}$

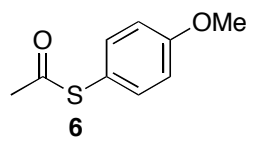

$\boldsymbol{S}$-(4-Methoxy)phenyl thioacetate (6). Flash chromatography over silica gel, using 10:90 EtOAc-hexanes gave $6(2.1141 \mathrm{~g} ; 93 \%)$ as a pure, yellow oil. Spectroscopic data was identical to that reported previously. ${ }^{1}$

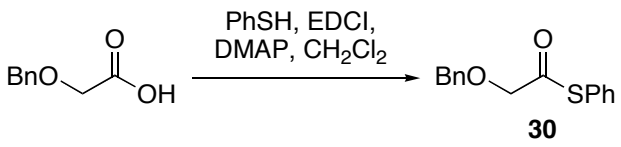

$S$-Phenyl benzyloxythioacetate (30). EDCI (1.3073 g, $6.82 \mathrm{mmol})$ and DMAP (0.0731 $\mathrm{g}$; $0.60 \mathrm{mmol})$ were added to a stirred solution of benzyloxyacetic acid $(1.0323 \mathrm{~g}, 6.21$ $\mathrm{mmol})$ and PhSH $(0.95 \mathrm{~mL} ; 9.29 \mathrm{mmol})$. The mixture was allowed to stir for $3 \mathrm{~h}$ and was partitioned between EtOAc and water. The organic phase was washed with saturated aqueous $\mathrm{NaHCO}_{3}$, saturated aqueous $\mathrm{NaCl}$, dried $\left(\mathrm{MgSO}_{4}\right)$ and evaporated to give a colorless oil. Flash chromatography over silica gel, using 5:95 EtOAc-hexanes gave 30 (1.5081 g; 94\%) as a pure, colorless liquid. ${ }^{1} \mathbf{H}$ NMR $\left(\mathrm{CDCl}_{3}, 300 \mathrm{MHz}\right): \delta$ 7.48-7.29 $(\mathrm{m}, 10 \mathrm{H}), 4.73(\mathrm{~s}, 2 \mathrm{H}), 4.27(\mathrm{~s}, 2 \mathrm{H}) ;{ }^{13} \mathbf{C ~ N M R}\left(\mathrm{CDCl}_{3}, 300 \mathrm{MHz}\right): \delta 198.2,136.9,134.9$, 129.6, 129.3, 128.7, 128.3, 128.1, 75.0, 74.3; ESI-MS $m / z$ calcd for $\mathrm{C}_{15} \mathrm{H}_{14} \mathrm{NaO}_{2} \mathrm{~S}: 281.1$, found: 280.8 .

${ }^{1}$ Gulhane, R.; Chakraborti, A. K. Chem. Commun. 2003, 1896-1897. 


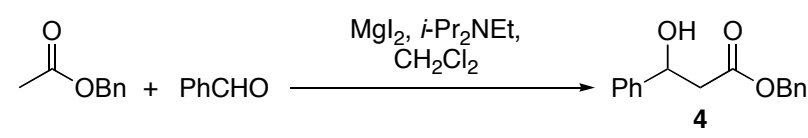

$\beta$-Hydroxy oxoester (4). $\mathrm{MgI}_{2}(0.167 \mathrm{~g}, 0.6 \mathrm{mmol})$ was added to a stirred solution of $O$ benzyl acetate (3) $(0.075 \mathrm{~g}, 0.5 \mathrm{mmol})$ and benzaldehyde $(61 \mu \mathrm{L}, 0.6 \mathrm{mmol})$ in $\mathrm{CH}_{2} \mathrm{Cl}_{2}$ $(2.5 \mathrm{~mL})$, followed by the addition of $i-\operatorname{Pr}_{2} \mathrm{NEt}(0.11 \mathrm{~mL}, 0.65 \mathrm{mmol})$. Stirring was continued for $20 \mathrm{~h}$ and EtOAc $(2.5 \mathrm{~mL})$ and $10 \%(\mathrm{v} / \mathrm{v})$ aqueous $\mathrm{HCl}(2.5 \mathrm{~mL})$ were added. Stirring was continued for 15 min and the mixture was partitioned between EtOAc $(15 \mathrm{~mL})$ and $\mathrm{H}_{2} \mathrm{O}(2 \mathrm{~mL})$. The aqueous phase was extracted with EtOAc $(3$ x 5 $\mathrm{mL}$ ) and the combined organic extracts were washed with saturated aqueous $\mathrm{NaCl}$, dried $\left(\mathrm{MgSO}_{4}\right)$, and evaporated. Flash chromatography over silica gel, using 10:90 EtOAchexanes gave $4(0.0593 \mathrm{~g} ; 46 \%)$ as a pure, colorless oil. Spectroscopic data was identical to that reported previously. ${ }^{2}$

\footnotetext{
${ }^{2}$ Wang, Y. C.; Hwang, J. Y.; Chen, Y. C.; Chuang, S. C.; Yan, T. H. Tetrahedron: Asymmetry 2000, 11, 1797-1800.
} 